\title{
Enumeration and Automatic Sequences
}

\author{
JEFFREY SHALLiT \\ School of Computer Science \\ University of Waterloo \\ Canada \\ email: shallit@cs.uwaterloo.ca
}

(Received: July 10, 2014, and in revised form July 2, 2015)

\begin{abstract}
A sequence $\left(a_{n}\right)_{n \geq 0}$ is $k$-automatic if there is a finite automaton that, on input $n$ expressed in base $k$, reaches a state with output $a_{n}$. In this paper I will survey some recent advances concerning enumeration of various aspects of these sequences, such as the recurrence function, and the subword complexity (which counts the number of distinct blocks of length $n$ ).
\end{abstract}

Mathematics Subject Classification(2010). 11B85, 68R15, 03D05, 68Q45, 11A63, $11 \mathrm{~A} 67$.

Keywords: enumeration, automatic sequence, recurrence, regular sequence, automaton, bordered word, synchronized sequence, predicate, logic, Fibonacci representation.

\section{Introduction}

Consider sequences over a finite alphabet $\Delta$. We know a lot about the properties of ultimately periodic sequences (those for which $a_{n+p}=a_{n}$ for all $n \geq N$ ) and about "random sequences". For example, consider subword complexity: the function $\rho(n)$ computing the number of distinct blocks ${ }^{1}$ of length $n$ appearing in the sequence. For ultimately periodic sequences we clearly have $\rho(n)=O(1)$, and in fact, this characterizes such sequences. For "random" sequences we have $\rho(n)=|\Delta|^{n}$ almost surely. Many of the "really interesting" sequences lie "in between" ultimate periodicity and total randomness.

One particular "in between" class that has been widely studied (e.g., 11, 12, 2]) is the class of $k$-automatic sequences. A sequence $\left(a_{n}\right)_{n \geq 0}$ is $k$-automatic if there is a finite automaton that, on input $n$ expressed in base $k$, reaches a state with output $a_{n}$. Classical examples of automatic sequences include the Thue-Morse sequence

$$
\mathbf{t}=0110100110010110 \cdots
$$

the Rudin-Shapiro sequence $\mathbf{r}=0001001000011101 \cdots$, the regular paperfolding sequence $\mathrm{p}=$ $0010011000110110 \cdots$, and many others.

For example, the Thue-Morse sequence is generated by the following automaton accepting $n$ expressed in base 2 . Here the output associated with each state is the name of the state.

\footnotetext{
${ }^{1}$ A block is a contiguous subsequence within a word. In the literature, blocks are sometimes called "factors", "subwords", or "subsequences". To make it more confusing, the latter two terms are sometimes used with other meanings.
}

DOI: $10.1515 /$ puma-2015-0008 


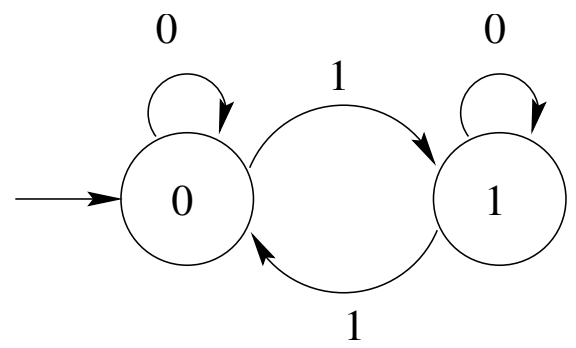

Very early on in the study of such sequences, researchers were interested in enumeration questions. For example, in 1938 Morse and Hedlund [21] computed the recurrence function $r_{\mathbf{t}}(n)$, which is the smallest block size that, no matter where the block appears, it is guaranteed to contain an occurrence of every length- $n$ block of $\mathbf{t}$. They showed that

$$
r_{\mathbf{t}}(n)= \begin{cases}3, & \text { if } n=1 \\ 9, & \text { if } n=2 \\ 9 \cdot 2^{j}+n-1, & \text { if } n \geq 3 \text { and } 2^{j}+2 \leq n \leq 2^{j+1}+1 .\end{cases}
$$

Brlek [5] (and independently, de Luca and Varricchio [14] and Avgustinovich [4]) computed the subword complexity of $\mathbf{t}$ :

$$
\rho_{\mathbf{t}}(n)= \begin{cases}2, & \text { if } n=1 ; \\ 4, & \text { if } n=2 ; \\ 4 n-4-2^{j}, & \text { if } n \geq 3 \text { and } 2^{j}+1 \leq n \leq 3 \cdot 2^{j-1}+1 \\ 2 n-2+2^{j+1}, & \text { if } n \geq 4 \text { and } 3 \cdot 2^{j-1}+1 \leq n \leq 2^{j+1}+1 .\end{cases}
$$

D'Alonzo [13] studied the repetitivity index of $\mathbf{t}$ : the minimum distance $d_{\mathbf{t}}(n)$ between any two consecutive occurrences of the same length- $n$ block. He showed that

$$
d_{\mathbf{t}}(n)= \begin{cases}1, & \text { if } n=1 \\ 2, & \text { if } n=2 ; \\ 3 \cdot 2^{j-1}, & \text { if } n \geq 3 \text { and } 2^{j}+1 \leq n \leq 3 \cdot 2^{j-1} \\ 2^{j+1}, & \text { if } n \geq 4 \text { and } 3 \cdot 2^{j-1}+1 \leq n \leq 2^{j+1} .\end{cases}
$$

Allouche and the author [2, §10.10] computed the appearance function $\alpha_{\mathbf{t}}(n)$ of the Thue-Morse sequence - the length of the shortest prefix containing every length- $n$ block appearing in $\mathbf{t}$ :

$$
\alpha_{\mathbf{t}}(n)= \begin{cases}2, & \text { if } n=1 \\ 7, & \text { if } n=2 \\ 6 \cdot 2^{j}+n-1, & \text { if } n \geq 3 \text { and } 2^{j}+2 \leq n \leq 2^{j+1}+1 .\end{cases}
$$

Goč, Henshall, and the author [16] computed the condensation function $C_{\mathbf{t}}(n)$ of the Thue-Morse sequence - the length of the shortest possible block of $\mathbf{t}$ containing every length- $n$ block appearing in 
t:

$$
C_{\mathbf{t}}(n)= \begin{cases}2, & \text { if } n=1 \\ 5, & \text { if } n=2 \\ 2^{j+2}+2 n-2, & \text { if } n \geq 3 \text { and } 2^{j}+2 \leq n \leq 2^{j+1}+1 .\end{cases}
$$

What these five measures have in common is that they can all be expressed in terms of the binary representation of $n$. This is not a coincidence. As it turns out, these and many other functions $f$ of $k$-automatic sequences are $k$-regular [1, 3]. This means that there is a linear representation of the sequence, i.e., there are vectors $v, w$ and a matrix-valued morphism $\mu: \Sigma_{k}^{*} \rightarrow \mathbb{Z}^{r \times r}$ such that

$$
f(n)=v \cdot \mu\left((n)_{k}\right) \cdot w^{T},
$$

where $(n)_{k}$ denotes the base- $k$ representation of $n$. Among other things, this implies that $f(n)$ can be computed in time polynomial in $\log n$.

Here is an example of a 2-regular sequence: the sequence $s_{2}(n)$, which is the sum of the bits in the binary expansion of $n$. Its linear representation is given by

$$
\begin{aligned}
v & =\left[\begin{array}{ll}
1 & 0
\end{array}\right] \\
\mu(0) & =\left[\begin{array}{ll}
1 & 0 \\
0 & 1
\end{array}\right] \\
\mu(1) & =\left[\begin{array}{cc}
0 & 1 \\
-1 & 2
\end{array}\right] \\
w^{T} & =\left[\begin{array}{ll}
0 & 1
\end{array}\right]
\end{aligned}
$$

\section{The enumeration procedure}

Here we sketch a procedure that allows one, in a purely mechanical way, to produce enumerations of the functions previously mentioned and many others. This gives a unified solution paradigm for many problems in the literature for which a number of ad hoc methods were employed previously.

There are four steps:

1. express the function in a certain logical theory;

2. translate the logical expression to an automaton;

3. convert the automaton to a linear representation like that in Eq. (1);

4. find a recurrence from the linear representation.

\subsection{The logical theory}

The logical theory we work with is $\operatorname{Th}\left(\mathbb{N},+, V_{k}\right)$, where $V_{k}(n)$ is the largest power of $k$ dividing $n$ ( $n o t$ the exponent of the largest power). As is well-known, this theory is decidable, yet powerful enough to express inequalities and automata [6]. It permits expressing predicates such as $a_{n}=b$, where $\mathbf{a}=\left(a_{n}\right)_{n \geq 0}$ is a $k$-automatic sequence. 
As an example, consider the predicate stating that the $k$-automatic sequence a contains a square, that is, two consecutive identical blocks (like the Italian word restereste). Letting $\mathbf{a}[i . . j]=a_{i} a_{i+1} \cdots a_{j}$, we can phrase the property of having a square as

$$
\exists i \geq 0 \exists n \geq 1 \mathbf{a}[i . . i+n-1]=\mathbf{a}[i+n . . i+2 n-1] .
$$

As stated, this is not quite in the right form, but it can be rewritten as

$$
\exists i \geq 0 \exists n \geq 1 \forall j(0 \leq j<n) \Longrightarrow \mathbf{a}[i+j]=\mathbf{a}[i+j+n]
$$

which is much closer.

For enumeration questions we need a predicate $P(n, i)$ of two variables. The intent is that our enumeration function $f(n)$ equals the number of distinct $i$ for which $P(n, i)$ is true. For example, if we want to count distinct blocks, each of which may occur many times in a sequence a, it suffices to express the assertion that the block at position $i$ is the first occurrence of that block. In this case we have

$$
P(n, i):=\forall j<i \mathbf{a}[i . . i+n-1] \neq \mathbf{a}[j . . j-n-1] .
$$

\subsection{Converting the expression to an automaton}

Now we have an expression in our logical theory. We parse the expression and employ the well-known automaton-based proof for the decidability of Presburger arithmetic [7. Numbers are represented in base $k$. Addition (more precisely, the assertion that $x+y=z$ ) can be represented by a 3 -state automaton. The statement " $\exists x P(x)$ " is implemented through guessing a nondeterministic choice of a base- $k$ representation of $x$, and " $\forall x P(x)$ " is implemented by rewriting it as " $\neg \exists x \neg P(x)$ ". Complementation is implemented by converting the (possibly nondeterministic) automaton to a deterministic one, and interchanging the roles of final and non-final states. This may require exponential blow-up in the number of states, in the worst case, but an implementation is nevertheless practical in many cases [16].

My graduate student Hamoon Mousavi has written a prover for this theory, called Walnut. It translates expression in the logical theory $\operatorname{Th}(\mathbb{N},+)$ supplemented with one or more automatic predicates to the corresponding automata. It is publicly available at

https://cs.uwaterloo.ca/〜shallit/Papers/Walnut.zip.

Let's use the Walnut prover to handle the predicate corresponding to subword complexity of the Thue-Morse sequence. We type

$$
\text { eval tmsc "Aj }(j<i) \Rightarrow(E k(k<n) \&(t[i+k] \quad !=t[j+k])) ":
$$

and get the following automaton as a result. It accepts pairs $(n, i)$ such that the length- $n$ block beginning at position $i$ occurs for the first time at that position. 


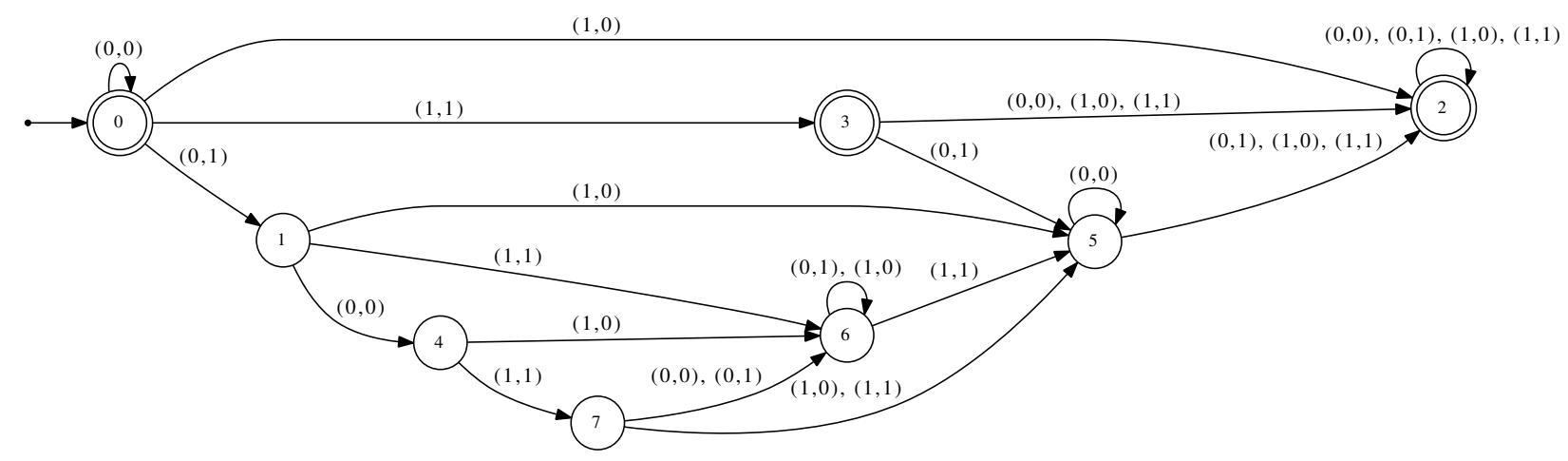

\subsection{Converting the automaton to a linear representation}

Once we have deterministic finite automaton $M=\left(Q, \Sigma, \delta, q_{0}, F\right)$ accepting the base- $k$ representation of pairs $\{(n, i): P(n, i)$ is true $\}$, we can easily convert it to a linear representation like Eq. (1) by setting

$$
(\mu(a))_{i, j}=\left|\left\{b: \delta\left(p_{i},[a, b]\right)=p_{j}\right\}\right|,
$$

where $p_{i}, p_{j}$ are states of $M$, and $\delta: Q \times \Sigma \rightarrow \Sigma$ is the transition function of $M$. Furthermore, $u, v$ are boolean vectors corresponding, respectively, to the initial and final states of $M$. Then the expression in Eq. (1) gives the number of $i$ corresponding to a given $n$. For more details, see [10].

\subsection{Finding a recurrence from the linear representation}

Finally, we can obtain a finite system of recurrences of the form

$$
f\left(k^{i} n+a\right)=\sum_{1 \leq j \leq r} c_{j} f\left(k^{i_{j}} n+a_{j}\right)
$$

through some simple linear algebra, as explained in [17].

We sketch the basic idea here. Let $(v, \mu, w)$ be a linear representation, and let $M$ be an arbitrary matrix matching the dimensions $d$ of $v$ and $w$. Let $v M=\left[a_{1}, a_{2}, \ldots a_{d}\right]$ where the $a_{i}$ are indeterminates. Now compute $v M \mu(y) w$ for words $y$; this will give an expression in terms of the indeterminates $a_{i}$. Then this gives a system of recurrences of the desired form.

\section{An example}

As an example, consider counting the number $u(n)$ of unbordered length- $n$ blocks of $\mathbf{t}$. A block is bordered if there is some nonempty nontrivial prefix that is also a suffix (like the Italian word rettore); otherwise it is unbordered.

The first step is to express the predicate $U(n, i):=$ the block $\mathbf{t}[i . . i+n-1]$ is unbordered. This can be done as follows:

$$
U(n, i):=\forall \ell \text { s.t. } 1 \leq \ell \leq n / 2 \mathbf{t}[i . . i+\ell-1] \neq \mathbf{t}[i+n-\ell . . i+n-1] .
$$


Thus we can express the predicate

$$
\begin{aligned}
P(n, i) & :=U(n, i) \text { and } \mathbf{t}[i . . i+n-1] \text { is the first occurrence of that block } \\
& =U(n, i) \text { and } \forall j<i \mathbf{t}[i . . i+n-1] \neq \mathbf{t}[j . . j+n-1],
\end{aligned}
$$

which has the property that $u(n)=\mid\{i: P(n, i)$ is true $\} \mid$.

Using the method sketched above, we can now find an automaton (in fact, it has 22 states) accepting the base- 2 representations of the pairs $\{(n, i): P(n, i)$ is true $\}$. We can then convert this to a linear representation, as follows:

$$
\begin{aligned}
& v=\left[\begin{array}{llllllllllllllllllllll}
0 & 0 & 0 & 1 & 0 & 0 & 0 & 0 & 0 & 1 & 0 & 0 & 0 & 0 & 0 & 0 & 1 & 0 & 0 & 0 & 0 & 0
\end{array}\right] \\
& {\left[\begin{array}{llllllllllllllllllllll}
0 & 0 & 1 & 0 & 0 & 0 & 1 & 0 & 0 & 0 & 0 & 0 & 0 & 0 & 0 & 0 & 0 & 0 & 0 & 0 & 0 & 0 \\
0 & 0 & 0 & 0 & 0 & 0 & 0 & 0 & 0 & 0 & 0 & 1 & 0 & 0 & 0 & 0 & 0 & 0 & 0 & 0 & 0 & 1
\end{array}\right]} \\
& \begin{array}{lllllllllllllllllllllll}
0 & 0 & 0 & 0 & 0 & 0 & 0 & 0 & 0 & 0 & 0 & 0 & 0 & 0 & 0 & 0 & 0 & 0 & 0 & 1 & 0 & 1
\end{array} \\
& \begin{array}{llllllllllllllllllllll}
0 & 0 & 0 & 0 & 0 & 0 & 0 & 0 & 0 & 0 & 0 & 0 & 0 & 0 & 0 & 0 & 1 & 0 & 0 & 0 & 0 & 0
\end{array} \\
& \begin{array}{llllllllllllllllllllll}
0 & 0 & 0 & 0 & 0 & 0 & 0 & 0 & 0 & 0 & 0 & 0 & 0 & 0 & 1 & 0 & 0 & 0 & 0 & 0 & 0 & 0
\end{array} \\
& \begin{array}{llllllllllllllllllllll}
0 & 0 & 0 & 0 & 0 & 0 & 0 & 0 & 0 & 0 & 0 & 0 & 1 & 0 & 0 & 0 & 0 & 0 & 0 & 0 & 1 & 0
\end{array} \\
& \begin{array}{llllllllllllllllllllll}
0 & 0 & 0 & 0 & 0 & 0 & 0 & 0 & 0 & 0 & 1 & 1 & 0 & 0 & 0 & 0 & 0 & 0 & 0 & 0 & 0 & 0
\end{array} \\
& \begin{array}{lllllllllllllllllllllll}
0 & 0 & 0 & 0 & 0 & 0 & 0 & 0 & 1 & 0 & 0 & 0 & 0 & 0 & 0 & 0 & 0 & 0 & 1 & 0 & 0 & 0
\end{array} \\
& \begin{array}{llllllllllllllllllllllllll}
0 & 0 & 0 & 0 & 0 & 0 & 0 & 0 & 0 & 0 & 0 & 0 & 1 & 0 & 0 & 0 & 0 & 0 & 0 & 0 & 1 & 0
\end{array} \\
& \begin{array}{lllllllllllllllllllllllll}
0 & 0 & 0 & 1 & 0 & 0 & 0 & 0 & 0 & 1 & 0 & 0 & 0 & 0 & 0 & 0 & 0 & 0 & 0 & 0 & 0 & 0 \\
0 & 0 & 0 & 0 & 0 & 0 & 0 & 0 & 0 & 0 & 0 & 0 & 0 & 0 & 0 & 1 & 0 & 0 & 0 & 0 & 0 & 0
\end{array} \\
& \mu(0)=\begin{array}{llllllllllllllllllllll}
0 & 0 & 0 & 0 & 0 & 0 & 0 & 0 & 0 & 0 & 0 & 0 & 0 & 0 & 0 & 1 & 0 & 0 & 0 & 0 & 0 & 0 \\
0 & 0 & 0 & 0 & 0 & 0 & 0 & 0 & 0 & 0 & 0 & 0 & 0 & 0 & 0 & 0 & 0 & 0 & 0 & 1 & 0 & 1
\end{array} \\
& \begin{array}{llllllllllllllllllllll}
0 & 0 & 0 & 0 & 0 & 1 & 0 & 0 & 0 & 0 & 0 & 0 & 0 & 0 & 0 & 0 & 0 & 0 & 0 & 0 & 1 & 0
\end{array} \\
& \begin{array}{llllllllllllllllllllll}
0 & 0 & 0 & 0 & 0 & 0 & 0 & 0 & 0 & 0 & 1 & 0 & 0 & 0 & 0 & 0 & 0 & 0 & 0 & 0 & 0 & 0
\end{array} \\
& \begin{array}{llllllllllllllllllllll}
0 & 0 & 0 & 0 & 1 & 0 & 0 & 0 & 0 & 0 & 0 & 0 & 0 & 0 & 0 & 0 & 0 & 0 & 0 & 0 & 0 & 0
\end{array} \\
& \begin{array}{llllllllllllllllllllll}
0 & 0 & 0 & 0 & 0 & 0 & 0 & 0 & 0 & 0 & 1 & 0 & 0 & 0 & 0 & 0 & 0 & 0 & 0 & 0 & 0 & 0
\end{array} \\
& \begin{array}{llllllllllllllllllllll}
0 & 0 & 0 & 0 & 0 & 0 & 0 & 0 & 0 & 0 & 0 & 0 & 0 & 0 & 0 & 0 & 0 & 0 & 0 & 0 & 0 & 0
\end{array} \\
& \begin{array}{llllllllllllllllllllll}
0 & 0 & 0 & 0 & 0 & 1 & 0 & 0 & 0 & 0 & 0 & 0 & 0 & 0 & 0 & 0 & 0 & 0 & 0 & 0 & 1 & 0
\end{array} \\
& \begin{array}{llllllllllllllllllllllllll}
0 & 0 & 0 & 0 & 0 & 0 & 0 & 0 & 0 & 0 & 0 & 0 & 0 & 0 & 0 & 0 & 0 & 0 & 1 & 0 & 1 & 0
\end{array} \\
& \begin{array}{llllllllllllllllllllll}
0 & 0 & 0 & 0 & 0 & 0 & 0 & 0 & 0 & 0 & 0 & 0 & 0 & 0 & 0 & 0 & 0 & 0 & 0 & 0 & 0 & 0
\end{array} \\
& {\left[\begin{array}{llllllllllllllllllllll}
0 & 0 & 0 & 0 & 0 & 0 & 0 & 0 & 0 & 0 & 0 & 0 & 0 & 0 & 0 & 0 & 0 & 0 & 1 & 0 & 1 & 0 \\
0 & 0 & 0 & 0 & 0 & 0 & 0 & 0 & 0 & 0 & 0 & 1 & 0 & 0 & 0 & 0 & 0 & 0 & 0 & 0 & 0 & 0
\end{array}\right]} \\
& \mu(1)=\left[\begin{array}{llllllllllllllllllllll}
0 & 0 & 0 & 0 & 0 & 0 & 0 & 0 & 0 & 0 & 0 & 0 & 1 & 0 & 0 & 0 & 0 & 0 & 1 & 0 & 0 & 0 \\
0 & 0 & 0 & 0 & 0 & 1 & 0 & 0 & 0 & 0 & 0 & 0 & 0 & 1 & 0 & 0 & 0 & 0 & 0 & 0 & 0 & 0 \\
0 & 0 & 0 & 0 & 0 & 0 & 0 & 0 & 1 & 0 & 0 & 0 & 0 & 1 & 0 & 0 & 0 & 0 & 0 & 0 & 0 & 0 \\
0 & 1 & 0 & 0 & 0 & 0 & 0 & 0 & 0 & 0 & 0 & 0 & 0 & 0 & 0 & 0 & 0 & 0 & 0 & 1 & 0 & 0 \\
0 & 0 & 0 & 0 & 0 & 0 & 0 & 0 & 0 & 0 & 1 & 0 & 0 & 0 & 0 & 0 & 0 & 0 & 0 & 0 & 1 & 0 \\
0 & 0 & 0 & 0 & 0 & 0 & 1 & 0 & 0 & 0 & 0 & 0 & 0 & 0 & 0 & 0 & 0 & 0 & 0 & 1 & 0 & 0 \\
0 & 0 & 0 & 0 & 0 & 1 & 0 & 0 & 0 & 0 & 0 & 1 & 0 & 0 & 0 & 0 & 0 & 0 & 0 & 0 & 0 & 0 \\
0 & 0 & 0 & 0 & 0 & 0 & 0 & 0 & 0 & 0 & 1 & 0 & 0 & 0 & 0 & 0 & 0 & 0 & 0 & 0 & 1 & 0 \\
0 & 0 & 0 & 0 & 0 & 0 & 1 & 0 & 0 & 0 & 0 & 0 & 0 & 0 & 0 & 0 & 0 & 0 & 0 & 1 & 0 & 0 \\
1 & 0 & 0 & 0 & 0 & 0 & 0 & 1 & 0 & 0 & 0 & 0 & 0 & 0 & 0 & 0 & 0 & 0 & 0 & 0 & 0 & 0 \\
0 & 0 & 0 & 0 & 0 & 0 & 0 & 0 & 0 & 0 & 0 & 0 & 0 & 0 & 0 & 0 & 0 & 1 & 0 & 0 & 0 & 0 \\
0 & 0 & 0 & 0 & 0 & 0 & 0 & 0 & 1 & 0 & 0 & 0 & 0 & 1 & 0 & 0 & 0 & 0 & 0 & 0 & 0 & 0 \\
0 & 0 & 0 & 0 & 0 & 0 & 0 & 0 & 0 & 0 & 1 & 0 & 0 & 0 & 0 & 0 & 0 & 0 & 0 & 0 & 1 & 0 \\
0 & 0 & 0 & 0 & 0 & 0 & 0 & 0 & 0 & 0 & 0 & 1 & 0 & 0 & 1 & 0 & 0 & 0 & 0 & 0 & 0 & 0 \\
0 & 0 & 0 & 0 & 0 & 0 & 1 & 0 & 0 & 0 & 0 & 0 & 0 & 0 & 0 & 0 & 0 & 0 & 0 & 1 & 0 & 0 \\
0 & 0 & 0 & 0 & 0 & 0 & 0 & 0 & 0 & 0 & 0 & 1 & 0 & 0 & 1 & 0 & 0 & 0 & 0 & 0 & 0 & 0 \\
0 & 0 & 0 & 0 & 1 & 0 & 0 & 0 & 0 & 0 & 1 & 0 & 0 & 0 & 0 & 0 & 0 & 0 & 0 & 0 & 0 & 0 \\
0 & 0 & 0 & 0 & 0 & 0 & 0 & 0 & 0 & 0 & 1 & 0 & 0 & 0 & 0 & 0 & 0 & 0 & 0 & 0 & 1 & 0 \\
0 & 0 & 0 & 0 & 0 & 0 & 0 & 0 & 0 & 0 & 0 & 0 & 0 & 0 & 0 & 0 & 0 & 0 & 0 & 0 & 0 & 0 \\
0 & 0 & 0 & 0 & 0 & 0 & 0 & 0 & 0 & 0 & 0 & 0 & 0 & 0 & 1 & 1 & 0 & 0 & 0 & 0 & 0 & 0 \\
0 & 0 & 0 & 0 & 0 & 0 & 0 & 0 & 0 & 0 & 0 & 0 & 0 & 0 & 0 & 0 & 0 & 0 & 0 & 0 & 0 & 0 \\
0 & 0 & 0 & 0 & 0 & 0 & 0 & 0 & 0 & 0 & 0 & 0 & 0 & 0 & 0 & 0 & 0 & 0 & 1 & 0 & 0 & 0
\end{array}\right]
\end{aligned}
$$


From this we can derive the following recurrence relations:

$$
\begin{array}{rlrl}
u(4 n) & =2 u(2 n), \quad(n \geq 2) & \\
u(4 n+1) & =u(2 n+1), \quad(n \geq 0) & & \\
u(8 n+2) & =u(2 n+1)+u(4 n+3), & (n \geq 1) & \\
u(8 n+3) & =-u(2 n+1)+u(4 n+2) & (n \geq 2) & \\
u(8 n+6) & =-u(2 n+1)+u(4 n+2)+u(4 n+3) & (n \geq 2) \\
u(8 n+7) & =2 u(2 n+1)+u(4 n+3) & (n \geq 3) &
\end{array}
$$

From this it is not hard [17] to deduce that, for example,

Theorem 3.1 We have $u(n) \leq n$ for $n \geq 4$. Furthermore, $u(n)=n$ infinitely often. Thus, $\lim \sup _{n \geq 1} u(n) / n=1$.

\section{Synchronized sequences}

The $k$-synchronized sequences form an interesting subset of the $k$-regular sequences. A function $f$ is $k$-synchronized 8 if there is an automaton that operates on two strings in parallel, and accepts those strings representing, in base $k$, the pair $(f(n), n)$. In this case, $f(n)$ can be computed in linear time $($ in $\log n)$ [18].

It is not hard to see that four of the five enumeration functions $f$ introduced above in Section 1 are $k$-synchronized. To do so, it suffices to give a predicate of the form $P(f, n)$ for them.

recurrence function:

$$
\begin{aligned}
& P(f, n)=\forall i \geq 0 \forall j \geq 0 \exists t \text { s.t. } i \leq t \leq i+f-n \text { s.t. } \mathbf{a}[j . . j+n-1]=\mathbf{a}[t . . t+n-1] \text { and } \\
& \exists i^{\prime} \geq 0, j^{\prime} \geq 0 \forall t^{\prime} \text { s.t. } i^{\prime} \leq t^{\prime} \leq i^{\prime}+f-n-1 \mathbf{a}\left[j^{\prime} . . j^{\prime}+n-1\right] \neq \mathbf{a}\left[t^{\prime} . . t^{\prime}+n-1\right] .
\end{aligned}
$$

Here the first part of the predicate specifies that there is a block of size $f$ that contains every length- $n$ block somewhere within it, and the second part of the predicate specifies that, for every block of size $f-1$, there is some length- $n$ block missing.

repetitivity index:

$$
\begin{aligned}
& P(f, n)=\exists i \geq 0 \text { s.t. } \mathbf{a}[i . . i+n-1]=\mathbf{a}[i+f . . i+f+n-1] \text { and } \\
& \qquad \forall i^{\prime} \geq 0, \forall j^{\prime}>i^{\prime}\left(\mathbf{a}\left[i^{\prime} . . i^{\prime}+n-1\right]=\mathbf{a}\left[j^{\prime} . . j^{\prime}+n-1\right]\right) \Longrightarrow j^{\prime} \geq i^{\prime}+f .
\end{aligned}
$$

Here the first part of the predicate specifies that there exists some length- $n$ block at distance $f$ from another occurrence, and the second part of the predicate specifies that no length- $n$ block has another occurrence closer than $f$.

appearance function:

$$
\begin{aligned}
& P(f, n)=\forall i \geq 0 \exists j \text { s.t. } 0 \leq j \leq f-n \text { and } \mathbf{a}[i . . i+n-1]=\mathbf{a}[j . . j+n-1] \text { and } \\
& \forall i^{\prime} \geq 0\left(\mathbf{a}[f-n . . f-1]=\mathbf{a}\left[i^{\prime} . . i^{\prime}+n-1\right]\right) \Longrightarrow i^{\prime} \geq f-n .
\end{aligned}
$$


Here the first part of the predicate specifies that every length- $n$ block that occurs somewhere, occurs in the prefix $\mathbf{a}[0 . . f-1]$, while the second part of the predicate specifies that the last length- $n$ block of a prefix of length $f$ appears only once in that prefix.

condensation function:

$$
\begin{aligned}
P(f, n)=\exists i \geq 0 \forall j \geq 0 \exists i^{\prime} \text { s.t. } i \leq i^{\prime}<i+f \text { and } \mathbf{a}[j . . j+n-1]=\mathbf{a}\left[i^{\prime} . . i^{\prime}+n-1\right] \text { and } \\
\forall i^{\prime \prime} \geq 0 \exists l \geq 0 \forall t \text { s.t. } i^{\prime \prime} \leq t \leq i^{\prime \prime}+f-n-1 \text { and } \mathbf{a}[l . . l+n-1] \neq \mathbf{a}[t . . t+n-1] .
\end{aligned}
$$

Here the first part of the predicate specifies that there exists a position $i$ such that the block of size $f$ beginning at that position contains all length- $n$ blocks, and the second part of the predicate specifies that for all blocks of size $f-1$, some length- $n$ block is missing.

However, for subword complexity, such a predicate is much harder to obtain. However, we have recently shown [18]

TheOREM 4.1 For $k$-automatic sequences $\mathbf{a}$, the predicate " $P(f, n)=$ the number of length-n blocks in a is $f$ " is expressible in $\operatorname{Th}\left(\mathbb{N},+, V_{k}\right)$.

It follows that this predicate is also $k$-synchronized.

\section{Enumeration in Fibonacci representation}

Recently the author and co-authors extended these ideas to sequences that are automatic functions of their Fibonacci (or "Zeckendorf") representation. This representation is of the form $\sum_{1 \leq i \leq t} e_{i} F_{t+2-i}$, where $F_{0}=0, F_{1}=1$, and $F_{n}=F_{n-1}+F_{n-2}$ for $n \geq 2$. As stated these representations are not unique, but if we also impose the condition that $e_{i} e_{i+1}=0$ for all $i$, then uniqueness follows.

The prototypical example of an infinite sequence that is "Fibonacci-automatic" is the infinite Fibonacci word $\mathbf{f}=f_{0} f_{1} f_{2} \cdots=01001010 \cdots$, defined as the fixed point of the morphism $0 \rightarrow 01$ and $1 \rightarrow 0$.

We have been able to obtain a number of new results concerning enumeration of various kinds of blocks of $\mathbf{f}$. As an example, consider the finite Fibonacci word $X_{n}$ which is the prefix of length $F_{n}$ of f. Then we have [15]:

TheOREm 5.1 Let $C(n)$ denote the number of cube occurrences in the Fibonacci word $X_{n}$. Then for $n \geq 3$ we have

$$
C(n)=\left(d_{1} n+d_{2}\right) \alpha^{n}+\left(d_{3} n+d_{4}\right) \beta^{n}+d_{5} n+d_{6}
$$

where $\alpha=(1+\sqrt{5}) / 2$ and $\beta=(1-\sqrt{5}) / 2$ and

$$
\begin{aligned}
d_{1} & =\frac{3-\sqrt{5}}{10} & d_{2} & =\frac{17}{50} \sqrt{5}-\frac{3}{2} \\
d_{3} & =\frac{3+\sqrt{5}}{10} & d_{4} & =-\frac{17}{50} \sqrt{5}-\frac{3}{2} \\
d_{5} & =1 & d_{6} & =-1 .
\end{aligned}
$$




\section{Open problems}

Not all functions of automatic sequences can be enumerated using the techniques we have described here. For example, Schaeffer [22] has shown that the predicate "a $[i . . i+2 n-1]$ is an abelian square" is not, in general, expressible in the logical theory $\operatorname{Th}\left(\mathbb{N},+, V_{k}\right)$.

Open Problem 6.1 Suppose $\mathbf{x}$ is a $k$-automatic sequence. Must the abelian complexity function $a_{\mathbf{x}}(n)$ (the number of distinct length-n blocks of $\mathbf{x}$, modulo abelian equivalence) be k-regular? This is true, for example, for the paperfolding sequence [20].

Cassaigne [9] introduced the notion of grouped factors. A sequence $\mathbf{a}=\left(a_{i}\right)_{i \geq 0}$ has grouped factors if, for all $n \geq 1$, there exists some position $m=m(n)$ such that $\mathbf{a}[m . . m+\rho(n)+n-2]$ contains all the $\rho(n)$ length- $n$ blocks of $\mathbf{a}$, each block occurring exactly once. One consequence of his result is that the Fibonacci word has grouped factors.

We can write a predicate for the property of having grouped factors, as follows:

$$
\begin{aligned}
\forall n \geq 1 \quad \exists m, s \geq 0 \quad \forall i \geq 0 \\
\quad \exists j \text { s.t. } m \leq j \leq m+s \text { and } \mathbf{a}[i . . i+n-1]=\mathbf{a}[j . . j+n-1] \text { and } \\
\quad \forall j^{\prime}, m \leq j^{\prime} \leq m+s, \quad j \neq j^{\prime} \text { we have } \mathbf{a}[i . . i+n-1] \neq \mathbf{a}\left[j^{\prime} . . j^{\prime}+n-1\right] .
\end{aligned}
$$

The first part of the predicate says that every length-n block appears somewhere in the desired window, and the second says that it appears exactly once.

(This five-quantifier definition can be viewed as a response to the question of Homer and Selman [19], "...in what sense would a problem that required at least three alternating quantifiers to describe be natural?")

Using this predicate and our decision method, we verified that the Fibonacci word does indeed have grouped factors.

Open Problem 6.2 Is there an aperiodic automatic sequence with grouped factors?

A weaker property results from changing "for all $n \geq 1$ " to "for infinitely many $n$ " in the definition above. This is also decidable, and results in

TheOREM 6.3 The Thue-Morse sequence has grouped factors exactly for $n=1$ and $n=2^{j}+1, j \geq 0$.

It would be nice to extend these ideas to larger classes of sequences. However, we cannot extend them to all morphic sequences, as the following example shows.

Theorem 6.4 Consider the morphism $h$ defined by $2 \rightarrow 2100,1 \rightarrow 100$, and $0 \rightarrow 0$. Let $\mathbf{a}=h^{\omega}(2)=$ $a_{0} a_{1} a_{2} \cdots$. Let $p$ be the morphic predicate that is true if $a_{n}=2$ or 1 , and false otherwise. Then the first-order theory $\operatorname{Th}(\mathbb{N},+,<, p)$ is undecidable.

Proof. It is easy to see that $\mathbf{a}=a_{0} a_{1} a_{2} \cdots$ is the characteristic word of the squares (modulo the fact that $\left.a_{0}=2\right)$. In the first-order theory $\operatorname{Th}(\mathbb{N},+,<, p)$ we can express a predicate $q(x, y)$ that is true if $y=x^{2}$ and false otherwise:

$$
q(x, y):=p(y) \wedge p(y+2 x+1) \wedge \forall t(p(t) \Longrightarrow((t \leq y) \vee(t \geq y+2 x+1))) .
$$


Suppose the predicate is true. Then $y$ is a square, say $y=u^{2}$, and $y+2 x+1$ is a square, say $y+2 x+1=v^{2}$, and there is no square strictly between $u^{2}$ and $v^{2}$. It follows that $v^{2}=(u+1)^{2}$ and so $u^{2}+2 x+1=y+2 x+1=v^{2}=(u+1)^{2}=u^{2}+2 u+1$. Hence $x=u$ and $y=x^{2}$. On the other hand, if $y=x^{2}$, then $p(y)$ is true and $p(y+2 x+1)=p\left(x^{2}+2 x+1\right)$ is true, and every square must be either $\leq x^{2}$ or $\geq(x+1)^{2}$. Once we have $q$ we can easily define a predicate $m(x, y, z)$ that is true if $z=x y$ and false otherwise:

$$
m(x, y, z):=\exists u \exists v \exists w p(u, x+y) \wedge p(v, x) \wedge p(w, y) \wedge(u=v+w+2 z) .
$$

Since it is well known that $\operatorname{Th}(\mathbb{N},+,<, \cdot)$ is undecidable [23], it follows that $\operatorname{Th}(\mathbb{N},+,<, p)$ is also undecidable.

\section{References}

[1] J.-P. Allouche And J. Shallit, The ring of k-regular sequences, Theoret. Comput. Sci., 98 (1992) 163-197.

[2] J.-P. Allouche And J. Shallit, Automatic Sequences: Theory, Applications, Generalizations, Cambridge University Press, 2003.

[3] J.-P. Allouche And J. Shallit The ring of k-regular sequences, II, Theoret. Comput. Sci., 307 (2003) 3-29.

[4] S. V. Avgustinovich, The number of different subwords of given length in the Morse-Hedlund sequence, Sibirsk. Zh. Issled. Oper., 1 (1994) 3-7. In Russian. English translation in A. D. Korshunov, ed., Discrete Analysis and Operations Research, Kluwer, 1996, pp. 1-5.

[5] S. BRLEK, Enumeration of factors in the Thue-Morse word, Discrete Appl. Math., 24 (1989) $83-96$.

[6] V. Bruyère, G. Hansel, C. Michaux and R. Villemaire, Logic and p-recognizable sets of integers, Bull. Belg. Math. Soc. Simon Stevin, 1 (1994) 191-238. Corrigendum, Bull. Belg. Math. Soc. Simon Stevin, 1 (1994) 577.

[7] J. R. BÜCHI, Weak second-order arithmetic and finite automata, Zeitschrift für mathematische Logik und Grundlagen der Mathematik, 6 (1960) 66-92. Reprinted in S. Mac Lane and D. Siefkes, eds., The Collected Works of J. Richard Büchi, Springer-Verlag, 1990, pp. 398-424

[8] A. CARPi And C. MAgGi, On synchronized sequences and their separators, RAIRO Theor. Inform. Appl., 35 (2001) 513-524.

[9] J. CASSAIGNE, Sequences with grouped factors, in Developments in Language Theory III, Aristotle University of Thessaloniki, 1998, 211-222.

[10] E. Charlier, N. Rampersad and J. Shallit, Enumeration and decidable properties of automatic sequences, Internat. J. Found. Comp. Sci., 23 (2012) 1035-1066. 
[11] A. CoBнam, On the base-dependence of sets of numbers recognizable by finite automata, Mathematical Systems Theory, 3 (1969) 186-192.

[12] A. Совнам, Uniform tag sequences, Mathematical Systems Theory, 6 (1972) 164-192.

[13] V. D'Alonzo, On the repetitivity index of infinite words, $\mathrm{PhD}$ thesis, Facoltà di Scienze Matematiche, Fisiche e Naturali, Università degli Studi di Napoli Federico II, 2009, available at http://www.fedoa. unina.it/3457/.

[14] A. De Luca And S. VarRicchio, Some combinatorial properties of the Thue-Morse sequence and a problem in semigroups, Theoret. Comput. Sci., 63 (1989) 333-348.

[15] C. F. Du, H. Mousavi, L. Schaeffer And J. Shallit, Decision Algorithms for Fibonacci-Automatic Words, with Applications to Pattern Avoidance, preprint, 2014, available at http://arxiv.org/abs/1406.0670.

[16] D. Goč, D. Henshall and J. Shallit, Automatic theorem-proving in combinatorics on words, Internat. J. Found. Comp. Sci., 24 (2013) 781-798.

[17] D. Goč, H. Mousavi and J. Shallit, On the number of unbordered factors, Lecture Notes in Comput. Sci., 7810 (2013) 299-310.

[18] D. Goč, L. Schaeffer and J. Shallit, Subword complexity and k-synchronization, Lecture Notes in Comput. Sci., 7907 (2013) 252-263.

[19] S. Homer And A. L. Selman, Computability and Complexity Theory, Springer-Verlag, 2nd edition, 2011.

[20] B. Madill and N. Rampersad, The abelian complexity of the paperfolding word, Discrete Math., 313 (2013) 831-838.

[21] M. Morse And G. A. Hedlund, Symbolic dynamics, Amer. J. Math., 60 (1938) 815-866.

[22] L. Schaeffer, Deciding properties of automatic sequences, Master's Thesis, University of Waterloo, 2013, https://uwspace. uwaterloo.ca/handle/10012/7899.

[23] A. TARski, Undecidable Theories, North-Holland, 2013. 